\title{
Detecção de Tubos em Imagens Radiográficas Digitais
}

\section{Pipe detection in digital radiographic images}

\author{
Márlon de Oliveira Vaz ${ }^{1}$ \\ Tania Mezzadri Centeno ${ }^{2}$ \\ Myriam Regattieri Delgado ${ }^{3}$
}

Data de submissão: 01/06/2015, Data de aceite: 15/02/2016

\begin{abstract}
Resumo: Este artigo apresenta uma metodologia para a detecção do tubo em imagens radiográficas do tipo parede dupla vista dupla (PDVD) de tubulações condutoras de petróleo. O principal objetivo da proposta é reduzir a região de busca através da delimitação da área do tubo para a extração automática do cordão de solda auxiliando, desta forma, a posterior detecção de defeitos em juntas soldadas. O processo de detecção do tubo apresentado é totalmente automático e baseado em técnicas de processamento de imagens como ajustes no brilho e contraste, limiarização e análise das regiões identificadas para a segmentação do tubo. O processo foi aplicado em 117 imagens provenientes de três diferentes sistemas radiográficos obtendo um resultado de $99,14 \%$ de acerto na detecção do tubo. Foi realizada uma comparação com outra abordagem para a detecção do tubo em imagens radiográficas do tipo PDVD e a metodologia proposta apresentou melhora em relação ao trabalho anterior. Concluise, portanto que o método proposto pode ser usado como uma etapa que precede a detecção automática do cordão de solda.
\end{abstract}

Palavras-chave: detecção de tubos, images radiográficas, PDVD, processamento digital de imagens

\footnotetext{
${ }^{1}$ Doutorando em Engenharia Elétrica e Informática Industrial / UTFPR, docente Instituto Federal do Paraná - IFPR - Curitiba - PR, Brasil. \{marlonvaz@gmail.com\}

${ }^{2}$ Doutorado em Doctorat En Informatique, docente Universidade Tecnológica Federal do Paraná - UTFPR - CuritibaPR, Brasil. \{mezzadri@utfpr.edu.br\}

${ }^{3}$ Doutorado em Engenharia Elétrica pela Universidade Estadual de Campinas, docente Universidade Federal do Paraná - UTFPR - Curitiba-PR, Brasil. \{myriamdelg@gmail.com\}
} 


\begin{abstract}
This paper presents a methodology to detect pipes in double wall double image (DWDI) radiographic images of petroleum pipelines. The main goal is to reduce the search region in automatic weld beads extractions (by delimiting the pipeline area), contributing this way to the subsequently detection of defects in welded joints. The pipe detection process is fully automatic and based on image processing techniques such as brightness and contrast adjustment, thresholding and analysis of the identified regions for pipe segmentation. Experiments consider 117 images acquired from three different radiographic systems with a result of $99,14 \%$ accuracy in the pipe detection. We also compare the detection results with those provided by a previous approach (developed to detect pipes in DWDI radiographic images) and the proposed methodology provides an improvement over previous work. We can conclude that the proposed method is a potential pre-processing step for detecting weld beads.
\end{abstract}

\title{
Keywords: pipe detection, radiographic images, DWDI, digital image processing
}

\section{Introdução}

Os ensaios não destrutivos (END) são amplamente utilizados pela indústria petroquímica para inspecionar soldas em tubos condutores de fluidos. Esta técnica permite ao inspetor avaliar a qualidade do cordão de solda sem causar impactos no sistema. Dentre as técnicas (END) mais utilizadas para análise da qualidade de um cordão de solda está o ensaio radiográfico. Esta técnica possibilita que uma imagem radiográfica seja gerada e posteriormente avaliada por um inspetor laudista qualificado. Além da vantagem do ensaio radiográfico poder ser utilizado na maioria das situações, este permite um registro permanente do exame realizado. Porém, a interpretação radiográfica não é tarefa simples, visto que o inspetor laudista é responsável por analisar um grande volume de imagens. Por ser uma tarefa exaustiva, os resultados podem variar quando se comparam laudos realizados por inspetores diferentes ou, até mesmo, no caso de laudos realizados pelo mesmo profissional sob diferentes circunstâncias no momento da execução da tarefa [1],[2].

Diante desse contexto e com base no desenvolvimento e aperfeiçoamento de equipamentos de radiografia digital, há atualmente uma grande quantidade de pesquisas em busca da automação parcial ou completa da interpretação radiográfica. Centros de pesquisa em diversos países têm focalizado seus esforços na tentativa de encontrar ferramentas computacionais automáticas que possam auxiliar na redução da subjetividade na interpretação radiográfica [3],[4],[5],[6],[7].

Uma área que tem avançado bastante é o estudo de técnicas de reconhecimento de padrões para classificação automática de defeitos de soldagem. Contudo, geralmente se recorre à extração manual ou semiautomática de regiões da imagem, denominadas regiões de inte- 
resse (ROI do inglês regions of interest), que no caso da aplicação considerada neste artigo, incluem o cordão de solda e os defeitos a serem analisados [5],[6],[7]. Para melhorar a eficiência dos métodos de detecção de defeitos seria conveniente utilizar métodos para segmentar a região do cordão de solda a ser inspecionado de forma a reduzir o espaço de busca. A detecção prévia do tubo evita que a etapa de busca pelo cordão de solda encontre outras regiões com características semelhantes às do cordão de solda. Além disso, o tempo computacional na busca pelo cordão é reduzido. Considerando os aspectos citados, surge a necessidade de se aprimorar as técnicas de segmentação de imagens radiográficas de juntas soldadas.

Assim, a detecção do cordão de solda é um passo fundamental para a automação de processos de inspeção por meio de imagens radiográficas. Na literatura encontram-se alguns trabalhos que tratam desse processo e que utilizam diferentes técnicas obtendo resultados diversos [2],[4],[8],[9],[10],[11], [12]. No entanto, somente os trabalhos de [4],[12],[13] e [14] tratam de cordões de solda do tipo parede dupla vista dupla (PDVD) que constituem a maioria das imagens encontradas no processo de inspeção de defeitos em cordões de solda com diâmetro igual ou inferior a 3 i polegadas. Em [4] e [12] a abordagem proposta utiliza uma detecção semiautomática do tubo, como etapa preliminar do processo de detecção do cordão de solda o que auxilia na identificação da região do cordão de solda.

Este artigo propõe um método automático para segmentar a região do tubo empregando técnicas de processamento digital de imagens, de forma que este possa ser utilizado como uma etapa anterior à detecção do cordão de solda. O trabalho apresenta, na seção 2 , o referencial teórico com conceitos básicos e trabalhos correlatos. A metodologia utilizada para resolver o problema de segmentação do tubo é detalhada na seção 3. Na seção 4 são apresentados os resultados obtidos com o método proposto e a seção 5 traz as conclusões do trabalho.

\section{Fundamentação Teórica}

Esta seção traz os conceitos básicos necessários para o entendimento da proposta. Na seção 2.1 a técnica de exposição radiográfica PDVD é apresentada de forma sucinta. A seção 2.2 discute as técnicas de processamento de imagens que serviram de base para a metodologia proposta. Por fim, na seção 2.3 , alguns trabalhos correlatos são apresentados.

\subsection{Técnica de exposição radiográfica PDVD}

A técnica de exposição radiográfica utilizada para a aquisição da imagem de cordões de solda está relacionada, principalmente com o tipo de montagem da tubulação e sua espessura. As tubulações com diâmetro igual ou inferior a 3 i polegadas $(90 \mathrm{~mm})$ não possibilitam acesso interno para a inserção da fonte de radiação, neste caso, a imagem radiográfica é usu- 
almente obtida através da técnica PDVD [3],[15].

Conforme ilustrado na figura 1, a técnica PDVD consiste em posicionar a fonte de radiação e o filme radiográfico no exterior do tubo, de forma que a radiação atravesse as duas paredes do tubo (parede dupla) e que as imagens das duas extremidades do cordão de solda sejam formadas no filme radiográfico (vista dupla). O cordão de solda é projetado no filme radiográfico apresentando um aspecto elíptico [15] A imagem da região do cordão de solda mais distante da fonte de radiação (parte superior do anel elíptico) se apresenta mais atenuada e também mais ruidosa, visto que a radiação atravessa as duas paredes da tubulação. Portanto, as abordagens usadas para tratar as imagens radiográficas provenientes dessa técnica devem considerar a falta de qualidade da imagem.

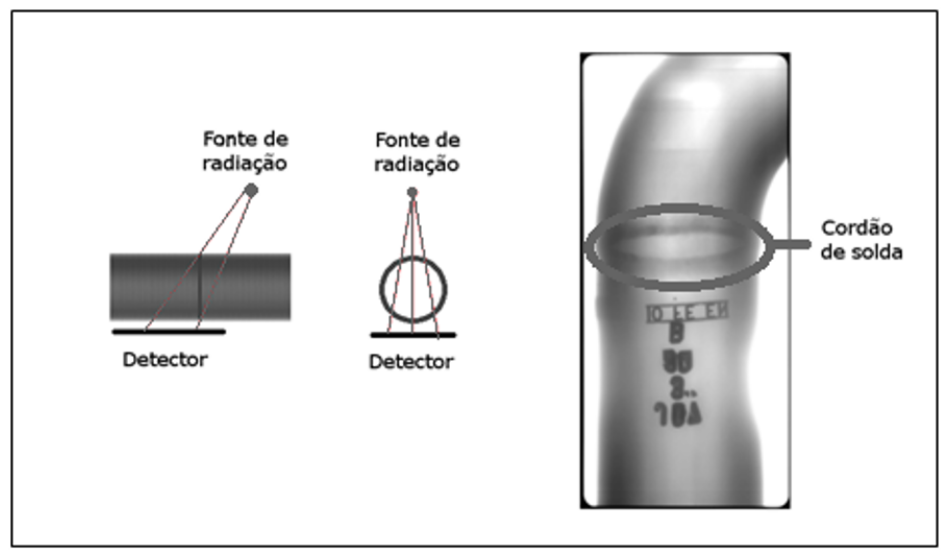

Figura 1. Técnica de exposição radiográfica PDVD com a imagem projetada do cordão de solda.

\subsection{Técnicas de processamento de imagens}

A região do cordão de solda a ser inspecionada (região delimitada pela elipse cinza escuro na Figura 1) constitui apenas uma pequena porção da imagem adquirida, na qual, além do tubo com marcações, aparece também a região do fundo. Desse modo, a localização prévia do cordão de solda é desejável na detecção automática de defeitos para otimizar o processo, limitando a região de busca. Entretanto, a localização automática do cordão não é trivial devido aos ruídos e pouco contraste nas bordas. Por isso a segmentação da região do tubo na qual o cordão está presente pode facilitar a busca. Diferentes técnicas de processamento digital de imagens podem ser empregadas para aprimorar a imagem e realçar as áreas de interesse facilitando a segmentação da região do tubo. 
Ajuste do contraste. Um dos problemas mais frequentemente encontrados em imagens digitais é o contraste e/ou brilho inadequado o que impede que as operações de segmentação sejam realizadas de forma eficiente. As técnicas de processamento de imagens usadas para realizar o ajuste do contraste são as transformações de intensidade [16]. Quando se deseja realçar uma faixa de níveis de cinza de forma a destacar o objeto de interesse na imagem uma transformação logarítmica pode ser empregada. A forma geral da curva logarítmica dessa transformação é definida por (1] [16]:

$$
s=c * \log (1+r)
$$

onde $r$ é o valor de nível de cinza do pixel atual $(r \geq 0)$, $s$ é o valor de nível de cinza do pixel mapeado e $c$ é uma constante escalar. A faixa dinâmica dependerá da intensidade da imagem podendo ser de $\left[0,2^{8}-1\right]$ ou de $\left[0,2^{16}-1\right]$. A transformação logarítmica é usada para expandir os valores de pixels mais baixos (mais escuros) em uma imagem e simultaneamente comprimir os valores de nível mais alto (mais claros).

Limiarização. Uma das formas mais simples de segmentar uma imagem é através da limiarização que transforma uma imagem em níveis de cinza em uma imagem binária com apenas dois níveis de cinza 0 (preto) e 1 (branco). Quando regiões de uma imagem (fundo e objetos) diferem entre si pela luminosidade (valores de nível de cinza), pixels pertencentes a diferentes regiões estarão em faixas limitadas por níveis de cinza distintos. Se estas faixas forem bem definidas é possível segmentar as regiões de interesse (objetos) através da limiarização [16]. A imagem segmentada $\mathrm{g}(\mathrm{x}, \mathrm{y})$ é obtida pela equação [2]:

$$
g(x, y)=\left\{\begin{array}{lll}
1 & \text { se } & f(x)>T \\
0 & \text { se } & f(x) \leq T
\end{array}\right.
$$

onde $f(x, y)$ corresponde ao nível de cinza do pixel da imagem de entrada localizado na coordenada $(x, y)$ e $T$ é um limiar, valor de nível de cinza que separa os pixels do objeto e do fundo. Este processo consiste em comparar os níveis de cinza dos pixels de uma imagem ao limiar $T$ e então classificá-los. Então qualquer ponto $(x, y)$ na imagem em que $f(x, y)>T$ é chamado de ponto do objeto; caso contrário, o ponto é chamado de ponto de fundo.

Na maioria das aplicações é importante que a determinação do limiar seja automática e para tal existem diversas técnicas. Sezgin e Sankur [17] avaliam 40 diferentes técnicas de limiarização aplicadas usualmente na área de END. Um dos métodos mais conhecidos de limiarização é o método de Otsu [18] que calcula o valor do limiar com base nas propriedades estatísticas da imagem.

Etiquetagem (Labeling). Na identificação de regiões o propósito é agrupar os pixels que possuem características semelhantes, como o nível de intensidade, criando assim, um conjunto de regiões [19]. Este processo pode ser aplicado para imagens binárias e imagens 
em nível de cinza. O algoritmo de etiquetagem atua em cada pixel da imagem verificado a sua vizinhança, caso ocorram valores de intensidade de similares, estes são conectados com o pixel inicial. Esta relação entre o ponto de origem e seus vizinhos é definida pelo tipo de conectividade. Neste caso, há dois modos de conectividade, a vizinhança-4 (four-neighbor) e a vizinhança-8 (eight-neighbor) [20]. Ao término do algoritmo cada agrupamento identificado é marcado numericamente possibilitando assim, definir as regiões dentro da imagem.

Neste trabalho foi utilizada a função bwlabel que faz parte do Image Processing Toolbox do Matlabß). Esta função foi desenvolvida com base no algoritmo run-length encoding [21]. O algoritmo percorre uma imagem binária linha a linha a procura pixels brancos, onde $\mathrm{P}$ é a quantidade de conjuntos de pixels contíguos encontrados em cada linha. Ao estabelecer uma sequência de pixels esta é armazenada em uma tabela contendo a linha, o pixel de início, o pixel de fim e a classificação $(P)$. Na execução da próxima linha são avaliados os pixels em relação à linha anterior determinado assim as adjacências entre os conjunto de pixels. Ao determinar a adjacência entre os grupos de pixels o algoritmo relaciona os conjuntos a uma tabela que expressa uma classe. Ao chegar à última linha o algoritmo organizou todos os conjuntos em classes.

Como já observado anteriormente, grande parte das pesquisas para a detecção de defeitos em cordão de solda realiza a extração manual da região de interesse, além disso, há poucas pesquisas que se concentram na técnica PDVD. Como será visto na próxima seção, nos trabalhos [4] e [12] a detecção do cordão de solda é realizada em dois passos: primeiramente é feita a detecção do tubo seguida da busca pelo cordão de solda no interior da imagem do tubo detectada. Entretanto, quando a imagem do tubo não é detectada, não é possível detectar o cordão de solda. Assim, este trabalho pretende contribuir nesta direção, propondo uma forma automática de detecção do tubo.

\subsection{Detecção do tubo/cordão de dolda em radiografias PDVD}

Como comentado anteriormente, a extração do cordão de solda na detecção automática de defeitos permite restringir o local da busca por descontinuidades no cordão. Essa restrição acelera a busca e evita que objetos que não estejam no interior da região desejada sejam detectados como defeitos (evita falsos positivos fora do cordão). Algumas abordagens se baseiam na segmentação da região do tubo como etapa preliminar, outras delimitam diretamente a região do cordão de solda.

Park et al. [22] apresentam uma técnica para segmentação da região do cordão de solda em imagens radiográficas do tipo PDVD pela extração dos perfis do tubo. Na etapa de pré-processamento é feita a remoção dos ruídos através da aplicação do filtro da média, o que reduz a intensidade dos picos no momento da extração dos perfis. Em seguida os perfis verticais são extraídos de três posições diferentes do tubo representando o fundo, o cordão de 
solda e a espessura do tubo. O perfil horizontal é extraído linha a linha, o qual em conjunto com os outros perfis estabelece os limites do tubo. A junção entre os perfis horizontais e verticais permite identificar a posição do cordão de solda. Os testes desenvolvidos pelos autores indicam que o método proposto pode ser aplicado às imagens radiográficas com baixo contraste, borrada e com ruídos.

Kroetz [4] e Kroetz et al. [12] realizam a detecção do cordão de solda em duas etapas. Primeiramente, a região do tubo é detectada e em seguida faz-se a busca pela região do cordão de solda dentro dos limites tubo. Em [4] e [12], a detecção do tubo utiliza técnicas bioinspiradas como algoritmos genéticos (AG) e otimização por enxame de partículas (PSO do inglês Particle Swarm Optimization) na produção de soluções candidatas. Estas soluções devem ser tais que maximizem a similaridade de um determinado recorte na imagem alvo em comparação com uma imagem modelo do tubo. O modelo do tubo é construído a partir de seis parâmetros que descrevem a posição do tubo na imagem como posições horizontal e vertical, largura, extensão, espessura e orientação. A partir desses atributos os algoritmos bioinspirados encontram a posição do tubo na imagem.

O trabalho desenvolvido em [12] utiliza imagens do tipo PDVD e obtém uma taxa de acerto no reconhecimento total do tubo de 75\% em 8 imagens. Em [4] o conjunto de imagens testadas é ampliado para 117 imagens do tipo PDVD e a abordagem apresenta como resultado final 20,51\% de acerto na detecção do tubo. Em [4] tal resultado influi diretamente na detecção do cordão de solda, pois se a imagem do tubo não é detectada corretamente, não é possível buscar a região do cordão de solda.

Suyama e colaboradores [13] e [14] apresentam uma técnica para auxiliar na localização do cordão de solda em imagens radiográficas do tipo PDVD, buscando a região central do cordão de solda. Os autores se baseiam no fato de que a região central do cordão de solda em imagens radiográficas do tipo PDVD tem formato elíptico. Inicialmente, são aplicadas técnicas de processamento de imagens para segmentar regiões (chamadas regiões candidatas) nas quais é possível inscrever uma elipse. Em seguida é usada uma técnica baseada em PSO para buscar a região que permite inscrever a elipse com a maior área possível. A região encontrada é definida como a região central do cordão de solda. Ambos os trabalhos usam nove imagens para a validação da técnica. Em [13], o percentual médio de acerto na classificação das regiões candidatas é de 77\%. Em [14], no qual se utiliza a técnica de PSO também para balancear os pesos dos parâmetros de busca, o percentual médio de acerto na detecção do cordão é de $88 \%$.

Embora as abordagens descritas em [13] e [14] busquem regiões elípticas na imagem que se assemelham à forma do cordão de solda, estas não são capazes de detectar apenas o cordão, mas apenas auxiliar na busca do cordão de solda. Os trabalhos apresentados em [13] e [14] não fazem a detecção prévia da região do tubo. 


\section{Metodologia Proposta}

Neste trabalho propõe-se uma nova técnica de segmentação automática do tubo cujas etapas do processo são ilustradas na figura 2 O algoritmo foi desenvolvido no software

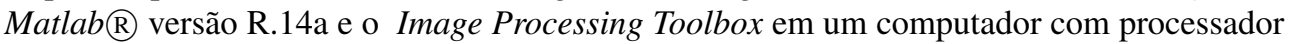
Core i7 e $8 \mathrm{~Gb}$ de memória.

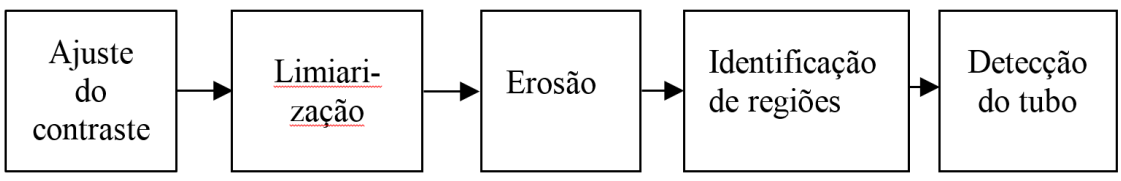

Figura 2. Processo de detecção do tubo.

A figura 3 (a) mostra uma imagem radiográfica do tipo PDVD contendo o tubo a ser segmentado. Observa-se que a região de fundo é constituída por uma parte escura contornada por um retângulo branco. O processo de detecção do tubo é realizado pelas etapas descritas a seguir.

Ajuste do contraste. Nesta etapa é aplicada a transformação logarítmica definida na equação (1). Essa transformação ajusta um intervalo expandindo os valores de intensidade mais baixos em um intervalo maior de níveis de saída com o objetivo de destacar a área do tubo do fundo da imagem. A figura 3 (c) apresenta a região do tubo de forma mais clara em relação à imagem original.

Limiarização. Após o ajuste do contraste, a imagem do tubo se torna mais nítida permitindo aplicar uma limiarização que converte a imagem para apenas dois níveis de cinza. A técnica de limiarização por Otsu [18] procura separar a imagem em duas classes distintas (Figura 3(e)). Observa-se que a limiarização não é suficiente para segmentar somente a área do tubo. Em algumas das imagens radiográficas, devido ao posicionamento do filme radiográfico, a região branca que deve pertencer ao fundo se conecta com a região do tubo como observado na parte inferior da figura 3 (b). Para minimizar os efeitos da limiarização por Otsu a imagem foi normalizada através da equação 3 que restringe faixas de intensidade para os valores desejados. Neste caso, às faixas com pequeno valor de intensidade são atribuídas o valor zero (preto), enquanto que as demais faixas próximas ao maior valor de intensidade passam a ter o valor máximo de intensidade da imagem ( $\max$ ). $\mathrm{O}$ valor $\max$ varia conforma a escala dinâmica da imagem podendo estar no intervalo $\left[0,2^{8}-1\right]$ ou no intervalo $\left[0,2^{16}-1\right]$ (Figura 3(d)).

$$
g(x, y)= \begin{cases}0 & \text { se } f(x, y) \geq 0.98 * \max \\ \max & \text { se } 0.2 * \max <f(x, y)<0.98 * \max \end{cases}
$$


Erosão. Para evitar a conectividade entre as regiões de fundo e do tubo e para reduzir pequenos objetos que possam interferir no processo de etiquetagem foi aplicada a erosão com um elemento estruturante (EE) retangular com tamanho 15x15, que além de remover as linhas finas reduziu a conexão entre regiões. Este operador morfológico permite que partes da imagem sejam reduzidas ou excluídas, como é o caso das linhas que aparecem na figura 3(e).

Identificação de regiões. Na imagem resultante, mesmo após o recorte, podem permanecer elementos indesejáveis que devem ser excluídos da segmentação final. Para tal aplicou-se um algoritmo de etiquetagem (labelling) que atribui um rótulo a cada região identificada como objeto na etapa anterior. A etiquetagem permite saber quantas regiões foram detectadas como objeto bem como a quantidade de pixels que cada região contém. Para a geração desta técnica foi utilizada a função bwlabel() do Image Processing Toolbox do $\operatorname{Matlab}{ }^{-}$.

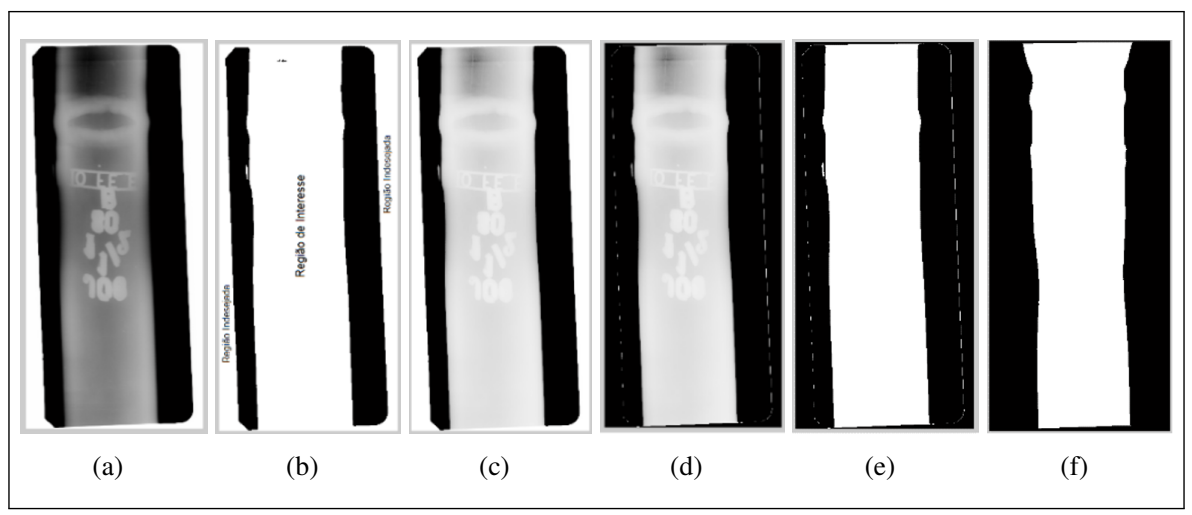

Figura 3. Resultado da aplicação das técnicas: (a) sobre a imagem original, (b) região de interesse, (c) ajuste do contraste, (d) limiarização por faixa dinâmica, (e) limiarização por OTSU e (e) erosão.

A figura 4 (b) mostra as regiões reconhecidas em cinza com contorno em branco. Dessa forma, é possível calcular a área de cada objeto detectado na imagem pela sua quantidade de pixels. O objeto com a maior área é designado como sendo a região do tubo, eliminando assim a informação irrelevante (figura 4(b)). O resultado final é a detecção do tubo em relação a imagem original (figura $4(\mathrm{c})$ ). 


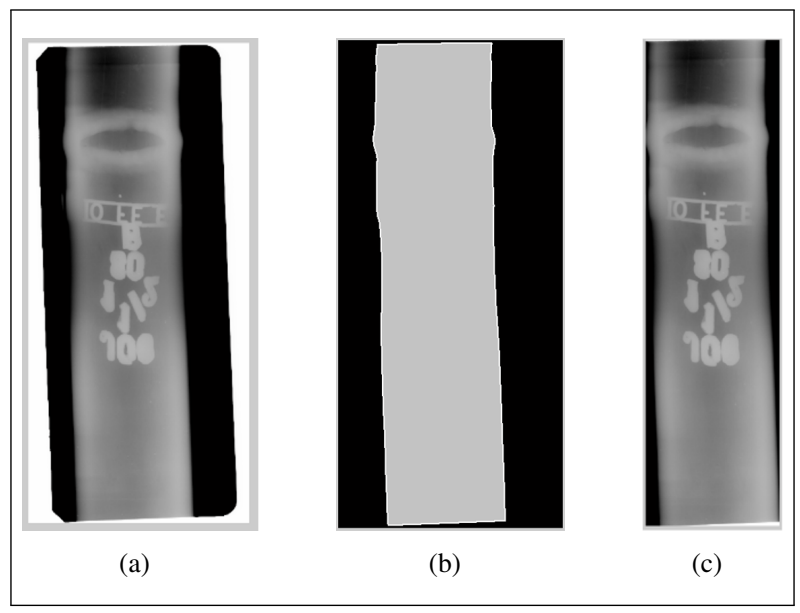

Figura 4. Processo de etiquetagem em a (a) Imagem original, (b) Etiquetagem e (c) Região do tubo detectado.

\section{Resultados e Discussões}

O método proposto neste trabalho foi aplicado sobre um conjunto de 117 imagens radiográficas digitais formato "TIFF", das quais 16 foram obtidas pelo sistema Dürr, 34 pelo sistema GE e 65 pelo sistema Kodak. As imagens Dürr possuem intensidade 16 bits e as demais 8 bits. O sistema proposto obteve neste conjunto de imagens uma precisão de $99,14 \%$ na identificação e extração do tubo. Todas as imagens do tudo estão dispostas verticalmente.

A qualidade das imagens utilizadas na base de dados varia de acordo com um conjunto de variáveis, entre elas o tipo de equipamento, tempo de exposição, filtros aplicados, tipo do filme, tipo do equipamento de digitalização, entre outros elementos [23]. Esses parâmetros influenciam não apenas no contraste da imagem como também na quantidade de ruído e na posição do tubo na imagem de forma que seria necessário considerar esses aspectos no algoritmo para que a porcentagem de acerto fosse maior.

A avaliação do método proposto levou em consideração o fato de que se o cordão de solda é mantido intacto na deteç̧ão do tubo o método atinge o objetivo e o resultado pode ser levado à próxima etapa, que é a detecção do cordão. Neste caso, o resultado é considerado como um acerto. Se a detecção do tubo perder o cordão de solda ou parte dele o resultado é insatisfatório, pois a etapa seguinte do processo não poderá detectar o cordão de solda. Todo o processo avaliativo foi executado através de análise visual envolvendo ambos os sistemas: Kroetz [4] e o método proposto. 
A figura 5(a) apresenta a imagem original, as demais imagens são as possíveis falhas na detecção do tubo, as quais foram recortadas manualmente para exemplificar os possíveis erros na detecção. Neste sentido, busca-se manter intacta a região do cordão de solda tanto no sentido vertical quanto horizontal. As figuras 5(b), 5(d), 5(e) e 5(f) mostram que parte do cordão de solda foi removido, enquanto que na figura 5 (c) a região do cordão foi extraída completamente.

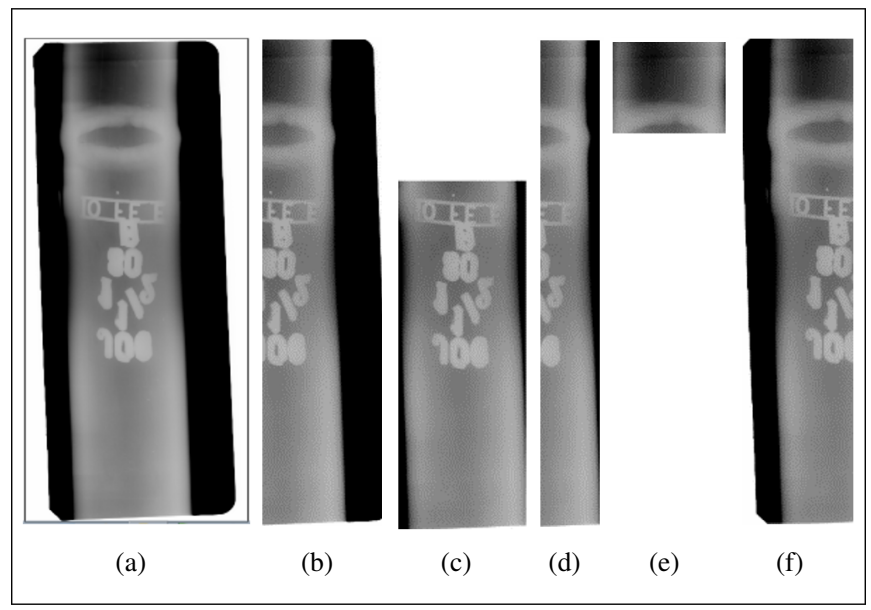

Figura 5. Imagens de referência com erros de detecção: (a) imagem original, (b), (c), (d), (e) e (f) erros de detecção.

Na figura 6 são apresentadas 4 imagens resultantes do processo de detecção do tubo através do sistema de Kroetz [4] relacionadas ao processo de avaliação utilizado. A figura 6(a) mostra uma detecção parcial do tubo com corte do cordão gerando um erro de detecção. Em 6.b) e 6(c) a região detectada é maior que a região do tubo, mas mantém a integridade do cordão de solda. A figura 6(d) apresenta a detecção ideal, ou seja, a região reconhecida equivale à área do tubo mantendo a região do cordão intacta.

Para uma melhor compreensão das diferenças entre os dois sistemas, a Figura 7 mostra o mesmo conjunto de imagens obtidos pelo sistema proposto após aplicação do mesmo sistema de avaliação visual. As imagens em 7(a), 7(b) e 7(c) detectam uma região maior que a área do tubo, mas mantém a região do cordão de solda, em 7 (d) tem-se a detecção ideal.

A figura 8 mostra uma falha na detecção do tubo pelo método proposto em relação ao método de Kroetz [4], pois a região do cordão de solda foi eliminada. O método de Kroetz [4] faz a detecção do tubo na mesma imagem mantendo a região do cordão de solda.

A metodologia proposta foi comparada com resultados obtidos pelo sistema de Kroetz 


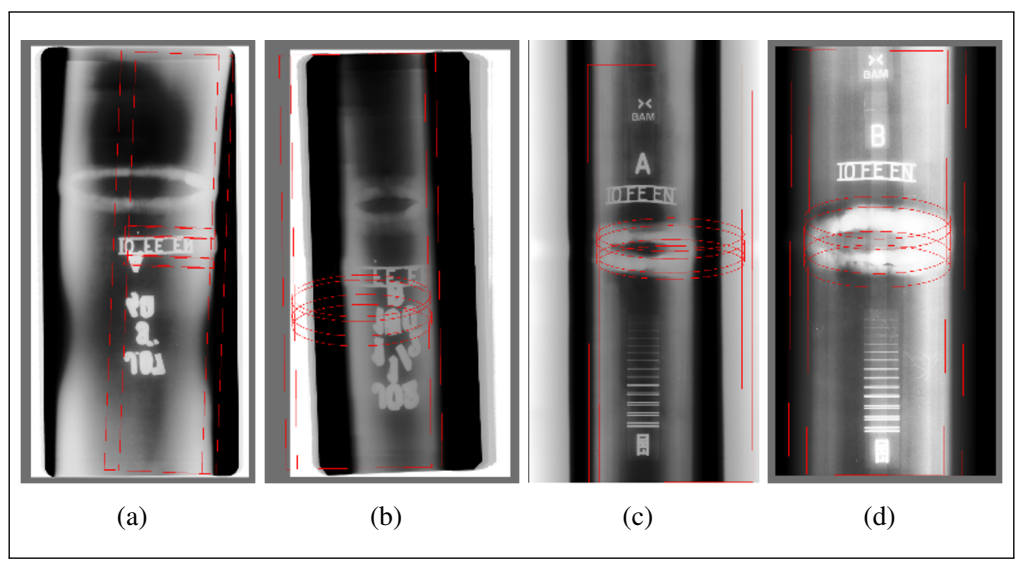

Figura 6. Imagens resultantes do processo visual para identificação da região do tubo pelo método de Kroetz [4]. (a) Erro de detecção, (b) detectado, (c) detectado e (d) detectado.

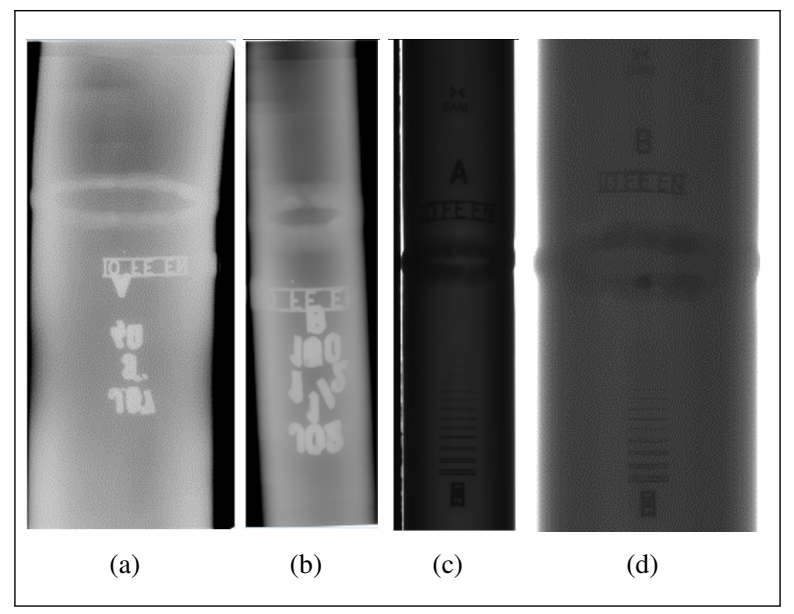

Figura 7. Imagens resultantes do processo visual para identificação da região do tubo pelo método proposto, em (a) detectado, (b) detectado, (c) detectado e (d) detectado.

[4] utilizando o mesmo conjunto de imagens. Neste caso, no geral o sistema de Kroetz [4] obteve uma precisão de $82,05 \%$ na detecção do tubo em relação ao conjunto total de imagens radiográficas, contra $99,14 \%$ do sistema proposto neste trabalho (Tabela 5). O sistema desenvolvido por Kroetz necessita de interação do usuário na etapa de pré-processamento 


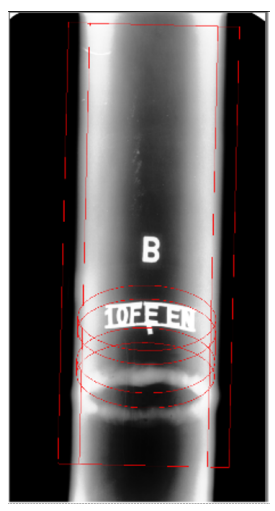

(a)

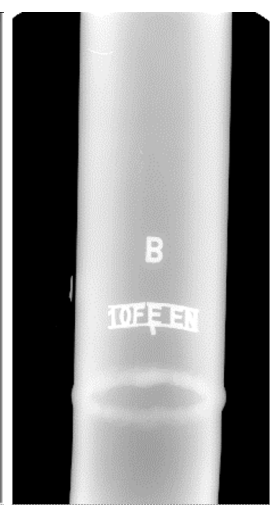

(b)

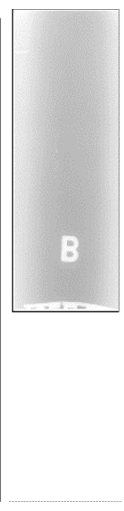

(c)

Figura 8. Resultados de detecção do tubo; (a) detecção pelo método Kroetz [4] avaliada como detectado, (b) imagem original para método proposto e (x) falha na detecção pelo método proposto.

e esta etapa depende do conhecimento prévio do usuário na seleção dos ajustes ideais. No teste foi utilizada a seguinte sequência de pré-processamento definida pelo autor [4]: inversão da imagem, equalização do histograma, aplicação do filtro da média com operador $3 \times 3$ para posteriormente executar a busca do tubo.

Para uma melhor compreensão dos resultados foram utilizadas 5 tabelas, onde as tabelas 1, 2 3 mostram respectivamente a quantidade de acertos para cada classe em relação aos sistemas Dürr, GE e Kodak. A tabela 4 apresenta os dados agrupados em relação à quantidade total de imagens utilizadas.

A tabela 5 apresenta o percentual para cada sistema e também o resultado total em relação ao conjunto de imagens utilizadas. Nesta tabela observa-se que o sistema proposto teve uma melhora significativa em relação ao método de Kroetz [4]. As tabelas mostram que o sistema proposto melhorou a detecção e extração da região do tubo, promovendo um avanço em relação à abordagem anterior.

\section{Conclusões}

A detecção do cordão de solda é uma etapa essencial para a realização de um processo automático de identificação de defeitos em imagens radiográficas de juntas soldadas de tu- 
Tabela 1. Classificação das imagens do sistema Dürr

\begin{tabular}{|l|c|c|}
\hline & Kroetz[4] & Sistema Proposto \\
\hline Erro & 4 & 0 \\
Detectado & 12 & 16 \\
total & 16 & 16 \\
\hline
\end{tabular}

Tabela 2. Classificação das imagens do sistema GE

\begin{tabular}{|l|c|c|}
\hline & Kroetz[4] & Sistema Proposto \\
\hline Erro & 4 & 0 \\
Detectado & 31 & 35 \\
total & 35 & 35 \\
\hline
\end{tabular}

Tabela 3. Classificação das imagens do sistema Kodak

\begin{tabular}{|l|c|c|}
\hline & Kroetz[4] & Sistema Proposto \\
\hline Erro & 13 & 1 \\
Detectado & 53 & 65 \\
total & 66 & 66 \\
\hline
\end{tabular}

Tabela 4. Classificação de todas as imagens de teste

\begin{tabular}{|l|c|c|}
\hline & Kroetz[4] & Sistema Proposto \\
\hline Erro & 21 & 1 \\
Detectado & 96 & 116 \\
total & 117 & 117 \\
\hline
\end{tabular}

Tabela 5. Taxa de detecção do tubo

\begin{tabular}{|l|c|c|}
\hline & Kroetz[4] & Sistema Proposto \\
\hline Dürr & $75 \%$ & $100 \%$ \\
GE & $88,57 \%$ & $100 \%$ \\
Kodak & $80,30 \%$ & $98,48 \%$ \\
total & $82,05 \%$ & $99,14 \%$ \\
\hline
\end{tabular}


bulações. Com o objetivo de reduzir o espaço de busca na detecção do cordão é desejável identificar previamente a posição do tubo. $\mathrm{O}$ método apresentado neste artigo utiliza uma abordagem simples e rápida para realizar a segmentação do tubo em imagens radiográficas de cordões de solda do tipo PDVD e pode ser utilizado como uma etapa anterior à detecção do cordão de solda.

O resultado alcançado foi de $99,14 \%$ no conjunto das imagens testadas (117 imagens) em função das amplas variações de contraste, da quantidade de ruído e do posicionamento do tubo, causadas por diferentes parâmetros no momento da aquisição e sistemas Dürr, GE e Kodak. Houve um avanço do método quando confrontado com o método proposto anteriormente por Kroetz [4] para o mesmo conjunto de imagens utilizadas como teste.

Em trabalhos futuros pretende-se incluir no algoritmo modificações que possam contemplar variações nas imagens decorrentes de diferentes ajustes de parâmetros na aquisição e incluir a etapa de segmentação do cordão de solda.

\section{Contribuição dos autores:}

- Marlon de Oliveira Vaz: concepção, planejamento, análise ou interpretação dos dados.

- Tania Mezzadri Centeno: revisão crítica de importante conteúdo intelectual e aprovação final da versão a ser publicada.

- Myrian Regattieri Delgado: revisão crítica de importante conteúdo intelectual.

\section{Referências}

[1] FüCSöK, F.; MULLER, C.; SCHARMACH, M. Human factors: the nde reliability of routine radiographic film evaluation. In: . c2000. v. 3.

[2] LIAO, T. W.; NI, J. An automated radiographic ndt system for weld inspection: part i weld extraction. Ndt \& E International, v. 29, n. 3, p. 157-162, 1996.

[3] FELISBERTO, M. K. Técnicas automáticas para detecção de cordões de solda e defeitos de soldagem em imagens radiográficas industriais. 2007. Tese (Doutorado em Engenharia Elétrica e Informática Industrial) - UTFPR, 2007.

[4] KROETZ, M. G. Sistema de apoio na inspeção radiográfica computadorizada de juntas soldadas de tubulações de petróleo. 2012. Dissertação (Mestrado em Engenharia Elétrica e Informática Industrial) - UTFPR, 2012. 
[5] ZAPATA, J.; VILAR, R.; RUIZ, R. Performance evaluation of an automatic inspection system of weld defects in radiographic images based on neuro-classifiers. Expert Systems with Applications, v. 38, n. 7, p. 8812-8824, 2011.

[6] XIAOMENG, W. Detection of weld line defect for oilgas pipeline based on X-rays image processing. In: . c2009. p. 273-275.

[7] HALIM, S. A.; HADI, N. A.; IBRAHIM, A.; MANURUNG, Y. H. The geometrical feature of weld defect in assessing digital radiographic image. In: . c2011. p. 189-193.

[8] LIAO, T. W.; TANG, K. Automated extraction of welds from digitized radiographic images based on mlp neural networks. Applied Artificial Intelligence, v. 11, n. 3, p. 197-218, 1997.

[9] LIAO, T.; LI, D.-M.; LI, Y.-M. Detection of welding flaws from radiographic images with fuzzy clustering methods. Fuzzy sets and Systems, v. 108, n. 2, p. 145-158, 1999.

[10] LIAO, T. W. Fuzzy reasoning based automatic inspection of radiographic welds: weld recognition. Journal of Intelligent Manufacturing, v. 15, n. 1, p. 69-85, 2004.

[11] FELISBERTO, M. K.; LOPES, H. S.; CENTENO, T. M.; DE ARRUDA, L. V. R. An object detection and recognition system for weld bead extraction from digital radiographs. Computer Vision and Image Understanding, v. 102, n. 3, p. 238-249, 2006.

[12] KROETZ, M.; CENTENO, T. M.; DELGADO, M. R.; FELISBERTO, M.; LUCAS, L. A.; DORINI, L. B.; FYLYK, V.; VIEIRA, A. Genetic algorithms to automatic weld bead detection in double wall double image digital radiographs. In: . c2012. p. 1-7.

[13] SUYAMA, F. M.; KREFER, A. G.; FARIA, A. R.; CENTENO, T. M. Identificação da região central de cordões de solda em imagens radiograficas de tubulações de petróleo do tipo parede dupla vista dupla. In: . c2013.

[14] SUYAMA, F. M.; KREFER, A. G.; FARIA, A. R.; CENTENO, T. M. Detecting central region in weld beads of dwdi radiographic images using pso. International Journal of Natural Computing Research (IJNCR), v. 5, n. 1, p. 42-56, 2015.

[15] BOILER, A.; CODE, P. V. Section v: Nondestructive examination. Subcommittee on Fiber-Reinforced Plastic Pressure Vessels, 2011.

[16] GONZALEZ, R. C.; WOODS, R. E. Processamento digital de imagens. tradução: Cristina yamagami e leonardo piamonte. Pearson Prentice Hall, São Paulo, 2010.

[17] SEZGIN, M. et al. Survey over image thresholding techniques and quantitative performance evaluation. Journal of Electronic imaging, v. 13, n. 1, p. 146-168, 2004. 
[18] OTSU, N. A threshold selection method from gray-level histograms. IEEE Transactions on Systems, Man and Cybernetics, v. 9, n. 1, p. 54-63, 1979.

[19] SHIH, F. Y. Image processing and pattern recognition: fundamentals and techniques. John Wiley \& Sons, 2010.

[20] NIXON, M. Feature extraction \& image processing. Academic Press, 2008.

[21] SHAPIRO, L.; HARALICK, R. Computer and robot vision. Addison-Wesley, 1992. v. I.

[22] PARK, S.-K.; AHN, Y.-S.; GIL, D.-S. The study on evaluation for digital radiography image of weldmentsthe study on evaluation for digital radiography image of weldments. In: . c2011.

[23] DA SILVA, R. R.; MERY, D. State-of-the-art of weld seam inspection by radiographic testing: part i-image processing. Materials Evaluation, v. 65, n. 6, p. 643-647, 2007. 